\title{
Bell Inequalities with Auxiliary Communication
}

\author{
D. Bacon* and B. F. Toner ${ }^{\dagger}$ \\ Institute for Quantum Information, California Institute of Technology, Pasadena, California 91125 \\ Department of Physics, California Institute of Technology, Pasadena, California 91125
}

(Received 11 August 2002; published 18 April 2003)

\begin{abstract}
What is the communication cost of simulating the correlations produced by quantum theory? We generalize Bell inequalities to the setting of local realistic theories augmented by a fixed amount of classical communication. Suppose two parties choose one of $M$ two-outcome measurements and exchange 1 bit of information. We present the complete set of inequalities for $M=2$, and the complete set of inequalities for the joint correlation observable for $M=3$. We find that correlations produced by quantum theory satisfy both of these sets of inequalities. One bit of communication is therefore sufficient to simulate quantum correlations in both of these scenarios.
\end{abstract}

DOI: 10.1103/PhysRevLett.90.157904

What are the differences between a quantum information processing device and its classical counterpart? The discovery that quantum computers can outperform classical computers [1] has made answering this question a central goal for the field of quantum information. Nearly 40 years ago, Bell [2] pointed out that the correlations resulting from quantum theory cannot be reproduced by any classical local realistic theory. It follows that quantum correlations on spacelike separated systems cannot be reproduced classically. If, however, the systems are timelike separated, then classical simulation is possible, albeit at the expense of some communication, but how much is required? In particular, suppose a number of spatially separate parties share an entangled quantum state, and each makes a local measurement on their component. Then quantum correlations are manifest in the joint probability distribution of the parties' outcomes, dependent on each party's choice of measurement. If this probability distribution cannot be reproduced by a classical local realistic theory, then it violates some generalized Bell inequality [3]. This means some communication between the parties is required to reproduce the probability distribution, but Bell inequality violation does nothing to quantify how much. More generally, entanglement is a resource for performing information processing tasks, and an important goal of quantum information theory is to demarcate it from classical resources, such as shared randomness and classical communication channels. What classical resources are required to reproduce the joint probability distributions arising from local measurement on shared quantum states?

We address the above question in this Letter. Within the setting of local realistic theories augmented by a fixed amount of two-way classical communication [4], we introduce the notion of Bell inequalities with auxiliary communication. These inequalities provide conditions on the joint probability distribution, which must be satisfied if such correlations can be simulated with shared randomness and a fixed amount of communication. Of
PACS numbers: 03.67.Mn, 03.65.Ta, 03.65.Ud, 03.67.Hk

particular significance are complete sets of such inequalities, which provide necessary and sufficient conditions. In the scenario where two parties choose one of $M$ twooutcome measurements and exchange 1 bit of information, we present the complete set of inequalities for $M=2$, and the complete set of inequalities for the joint correlation observable for $M=3$. We find that quantum correlations satisfy all of these inequalities, irrespective of the particular quantum state or the specific measurements, and can therefore be explained in these settings by augmenting a local realistic theory with a single bit of communication. This is particularly remarkable for the $M=3$ case, where one would naively expect a trit of auxiliary communication is required to simulate quantum correlations.

The model. - We restrict attention to scenarios with two parties, $A$ and $B$. In a measurement scenario for this bipartite case, each party selects one of $M$ different measurements and then - possibly after some delay, during which we might allow the parties to communicate outputs one of $K$ different outcomes. (Note that $A$ and $B$ may choose measurements from different $M$-element sets.) Such a measurement scenario results in a set of probabilities $0 \leq p_{a, b \mid i, j} \leq 1$, where $p_{a, b \mid i, j}$ is the probability that $A$ outputs $a$ and $B$ outputs $b$, given that $A$ selects measurement $i$ and $B$ selects measurement $j$. Discounting null outcomes (which can be incorporated as a separate outcome if desired), it follows that $\sum_{a=0}^{K-1} \sum_{b=0}^{K-1} p_{a, b \mid i, j}=1$, where $0 \leq i, j \leq M-1$. A valid measurement scenario is any set of probabilities which satisfies these normalization constraints.

Given a particular measurement scenario, we investigate all protocols which the two parties might perform to produce the correct probabilities. A protocol consists of three stages: (i) preparation via the distribution of shared randomness, (ii) communication via the exchange of messages between the parties, and (iii) output of outcomes by each party as determined by information accessible to each party. $A$ and $B$ select their measurements after step (i) but before step (ii). If a protocol produces identical 
probabilities to the measurement scenario, then we say that the protocol has simulated the scenario.

Two informational resources are of interest: the quantity of shared randomness and the amount of communication between the parties. We focus on the amount of communication and define the cost of a protocol to be the maximum amount of communication required (as opposed to the average amount of communication, see [5]). In the preparation phase of a protocol, we allow $A$ and $B$ to share an infinite amount of classical information and, in particular, continuous variables. In the parlance of foundational studies of quantum theory, these are known as local hidden variables (LHVs) [6]. A protocol with no communication [step (ii) missing] is usually called a $L H V$ theory. In such a theory, each party's output depends on the shared randomness and on which measurement the particular party has locally selected, but not on the measurement choice of the other party.

The protocols we investigate are therefore an extension of LHV theories, where we allow the parties to communicate after selecting measurements [4]. This allows some "which measurement" information to propagate between the parties. We emphasize that a protocol of this form simulates the joint probability distribution resulting from a set of quantum measurements, but not the quantum measurements themselves: it is not possible to replace local measurements made by two spacelike separated parties on an entangled quantum state by classical communication. Even in this case, however, the amount of two-way communication required to reproduce the joint probability distribution provides a measure of the nonlocality of the correlations. From an information processing perspective, this model provides a fair setting for the comparison of quantum correlations and classical resources required to reproduce them.

Of particular significance in this respect is the result of Brassard, Cleve, and Tapp [4], who demonstrated that the correlations produced by two-outcome projective measurements on an EPR pair can be simulated by a local realistic theory augmented by 8 bits of communication. Surprisingly, we have recently shown that a single bit of communication is sufficient [7].

Little, however, is known for more general states and more general measurements. The goal of this paper is to illuminate how such bounds can be achieved by generalizing Bell inequalities to what we term, Bell inequalities with auxiliary communication.

Bell polytopes. - Bell inequalities [2] describe necessary conditions on the probabilities $p_{a, b \mid i, j}$, which must be satisfied if these probabilities are to be produced by a local realistic theory. When a set of these conditions is also sufficient, we say that we have a complete set of Bell inequalities. The construction of complete sets of Bell inequalities is an exercise in convex geometry [8]. In this section, we briefly sketch the construction for Bell inequalities without auxiliary communication.
Consider a deterministic protocol, i.e., one in which no randomness, shared or otherwise, is used. [This corresponds to a protocol consisting only of step (iii) above, with the additional requirement that this step is deterministic.] Each party's output can depend only on their local which measurement information, so that all such protocols can be completely characterized by two functions $\alpha, \beta: \mathbb{Z}_{M} \rightarrow \mathbb{Z}_{K}$, which describe the outcomes of the two parties' measurements: if $A$ selects measurement $i$, she outputs $\alpha(i)$ and if $B$ selects measurement $j$, he outputs $\beta(j)$. The probabilities for the scenario are then $p_{a, b \mid i, j}=\delta_{\alpha(i)}^{a} \delta_{\beta(j)}^{b}$.

By listing the components, we may view the probabilities $p_{a, b \mid i, j}$ as vectors $\vec{p}$ in $\mathbb{R}^{D}$ with $D=M^{2}\left(K^{2}-1\right)$ (recall the constraint $\sum_{a, b} p_{a, b \mid i, j}=1$ ). To each pair of functions $\{\alpha, \beta\}$, there corresponds a deterministic protocol, so the set of all deterministic protocols is a finite collection of such vectors $\left\{\vec{d}_{\zeta} \mid \zeta=1, \ldots, K^{2 M}\right\}$.

Now consider the effect of allowing randomness. Any unshared randomness can always be replaced by shared randomness on which the other party does not act [9], so we may continue to assume step (iii) is deterministic. But then every set of random variables in step (i) corresponds to a particular deterministic protocol. Therefore the set of all possible protocols which use randomness and no communication is described by a convex sum of the deterministic protocols without communication,

$$
\vec{p}=\sum_{\zeta} \lambda_{\zeta} \vec{d}_{\zeta}, \quad \sum_{\zeta} \lambda_{\zeta}=1, \quad \lambda_{\zeta} \geq 0 .
$$

The set of all protocols therefore corresponds to a region $\Omega_{M K}$ in $\mathbb{R}^{D}$, which is a polytope because there are a finite number of extreme vectors $\vec{d}_{\zeta}$ [10]. This permits an alternative description: instead of describing the polytope $\Omega_{M K}$ as the convex combination of a finite set of extreme points, we can instead describe it by specifying a complete (finite) set of facet inequalities. A facet inequality is a pair $\{\vec{f}, c\}$ which defines a half-space of $\mathbb{R}^{D}$ via the inequality $\vec{f} \cdot \vec{p} \leq c$. Complete sets of facet inequalities $\left\{\vec{f}_{\eta}, c_{\eta}\right\}$ are satisfied if and only if $\vec{p}$ is in $\Omega_{M K}$ :

$$
\vec{p} \in \Omega_{M K} \quad \text { iff } \vec{f}_{\eta} \cdot \vec{p} \leq c_{\eta}, \forall \eta .
$$

Each facet is therefore a Bell inequality and complete sets of facet inequalities are complete sets of Bell inequalities. Complete sets are known in the two party case when $M=2, K=2$ [11], $M=3, K=2$ [12], and also when extra symmetry constraints are imposed [13].

Bell inequalities with auxiliary communication. - We now turn to the main focus of our Letter: extending the formalism of Bell inequalities to protocols which permit communication after the parties have chosen their measurements. Again consider a deterministic protocol, but now allow for the communication (possibly two-way) of at most $r$ bits of information between the parties after selection of measurements. Such a protocol is completely characterized by two functions $\alpha, \beta: \mathbb{Z}_{M} \otimes \mathbb{Z}_{M} \rightarrow$ $\mathbb{Z}_{K}$, which describe the outcomes of the two parties' 
measurements, but now each party's output can also depend on which measurement the other party selects: if $A$ selects measurement $i$ and $B$ measurement $j, A$ outputs $\alpha(i, j)$ and $B$ outputs $\beta(i, j)$. The probabilities for such a deterministic protocol are then $p_{a, b \mid i, j}=\delta_{\alpha(i, j)}^{a} \delta_{\beta(i, j)}^{b}$. While $\alpha$ and $\beta$ can now depend on which measurement the other party selects, not all functions $\alpha(i, j), \beta(i, j)$ are necessarily accessible, if the parties exchange at most $r$ bits of communication. The set of possible functions $\alpha(i, j), \beta(i, j)$ for protocols which use at most $r$ bits of communication is the subject of the field of communication complexity $[9,14]$. For example, with a single bit of communication from $A$ to $B, \alpha(i, j)$ is independent of $B$ 's measurement $j$ and $\beta(i, j)$ can depend only on a partition of the set of possible $i$ 's into two sets. Despite this complication, deterministic protocols still correspond to a finite set of vectors of probabilities $\vec{d}_{\zeta}^{(r)}$ in $\mathbb{R}^{D}$.

If we now allow randomness, the set of accessible probabilities $\Omega_{M K}^{(r)}$ is given by the convex combination of the deterministic probabilities, $\vec{p}=\sum_{\zeta} \lambda_{\zeta} \vec{d}_{\zeta}^{(r)}$, $\sum_{\zeta} \lambda_{\zeta}=1, \lambda_{\zeta} \geq 0$. Again, $\Omega_{M K}^{(r)}$ is a convex combination of a finite number of extreme points - a polytope - and can be described by a finite set of facet inequalities: $\vec{p} \in$ $\Omega_{M K}^{(r)}$ iff $\vec{f}_{\eta}^{(r)} \cdot \vec{p} \leq c_{\eta}, \forall \eta$. The complete set of facet inequalities for $\Omega_{M K}^{(r)}$ is a complete set of Bell inequalities with $r$ bits of communication. An important limit arises when $r \geq 2 \log _{2} M$ because then each party can tell the other exactly which measurement they have selected. In this setting, all deterministic protocols can be executed by the two parties: the probability distribution $p_{a, b \mid i, j}$ is unrestricted. This implies that Bell inequalities with auxiliary communication are trivial when $M=1$.

Additionally, for $r \geq \log _{2} M$, Bell inequalities with auxiliary communication, although not necessarily trivial, are never violated by probability distributions arising from local measurements on a shared quantum state. In fact this is true for any probability distribution satisfying the no-one-way-signaling conditions $[3], \quad p_{a \mid i, j} \equiv$ $\sum_{b=0}^{K-1} p_{a, b \mid i, j}=p_{a \mid i}$, is independent of $j$ for all $a$ and $i$ : $A$ 's marginal probability distribution is independent of $B$ 's choice of measurement. In such cases, it is sufficient that only $A$ communicate her measurement choice. The simulation procedure is as follows: for each of $A$ 's measurements $i$, the parties share a random variable $\tilde{a}_{i}$ drawn from the probability distribution $\left\{a, p_{a \mid i}\right\}$ (i.e., $\tilde{a}_{i}=a$ with probability $\left.p_{a \mid i}\right)$. Suppose $A$ chooses measurement $i$ and $B$ chooses measurement $j$. $A$ outputs $\tilde{a}_{i}$ and sends her measurement choice $i$ to $B$. $B$ then outputs $\tilde{b}_{\tilde{a}_{i}, i, j}$, where $\tilde{b}_{a, i, j}$ is drawn from the probability distribution $\left\{b, p_{\tilde{a}_{i}, b \mid i, j}\right\}$. (The roles of $A$ and $B$ in the no-one-waysignaling conditions and protocol may be reversed.)

A complete set of Bell inequalities with auxiliary communication.-Consider the simplest case $M=K=2$ and $r=1$ bit. The polytope $\Omega_{2,2}^{(1)}$ is 12 dimensional and has 112 extreme vectors. Using both the primal-dual algorithm and the double description method [15] for facet enumeration, we find that this polytope has 48 facets. 16 facets describe trivial inequalities, $p_{a, b \mid i, j} \geq 0$ $(0 \leq i, j, a, b \leq 1)$. Another 16 facets are of the form

$$
p_{a_{1}, b_{1} \mid 0,0}+p_{a_{2}, b_{2} \mid 0,1}+p_{a_{3}, b_{3} \mid 1,0}+p_{a_{4}, b_{4} \mid 1,1} \leq 2,
$$

with $\left(a_{1} a_{2} a_{3} a_{4}\right) \in\{(0101),(1010),(0110),(1001)\}$ and $\left(b_{1} b_{2} b_{3} b_{4}\right) \in\{(0011),(1100),(0110),(1001)\}$. The remaining 16 facets are given by

$$
p_{a, 0 \mid i, j}+p_{a, 1 \mid i, j}+p_{0, b \mid \bar{i}, \bar{j}}+p_{1, b \mid \bar{i}, \bar{j}}-p_{a, b \mid i, \bar{j}} \geq 0
$$

$(0 \leq i, j, a, b \leq 1)$ where $\overline{0}=1$ and $\overline{1}=0$. The above inequalities completely describe the region of probability distributions that can be created with 1 bit of communication. There are probability distributions which violate these inequalities: for example, if $p_{a, b \mid i, j}=\delta_{j}^{a} \delta_{i}^{b}$, Eq. (3) with $\left(a_{1} a_{2} a_{3} a_{4}\right)=(0101)$ and $\left(b_{1} b_{2} b_{3} b_{4}\right)=(0011)$ is maximally violated: substitution gives $4 \neq 2$.

It is straightforward to check that any probability distribution satisfying the no-signaling conditions satisfies inequalities Eqs. (3) and (4). Finally, consider the probability distribution $p_{a, b \mid i, j}=\frac{1}{2}\left(\delta_{0}^{a} \delta_{i}^{b}+\delta_{j}^{a} \delta_{0}^{b}\right)$. This probability distribution violates the no-signaling conditions (in both directions), but satisfies Eqs. (3) and (4), thus indicating that these inequalities are strictly stronger than no-signaling.

A complete set of Bell inequalities with auxiliary communication for the joint observable.-The above complete Bell inequalities with auxiliary communication were used to bound the allowed probabilities $p_{a, b \mid i, j}$ for protocols using a specified amount of communication. In quantum theory we are often interested not in all of the probabilities for a measurement scenario, but only on the value of a particular joint observable. This simplifies our computational task, because we may project the polytope $\Omega_{M K}^{(r)}$ onto a lower-dimensional subspace and only enumerate the facets of the projected polytope, as we shall explain in the following. We term a complete set of facet inequalities for this convex set a complete set of Bell joint observable inequalities with auxiliary communication.

Consider a measurement scenario with probabilities $p_{a, b \mid i, j}$ and identify measurement outcomes with values of local observables. The joint observable for the $i$ th and $j$ th measurements of $A$ and $B$ is then defined as

$$
c_{i, j}=\sum_{a=0}^{K-1} \sum_{b=0}^{K-1} A_{a} B_{b} p_{a, b \mid i, j},
$$

where $A_{a}$ and $B_{b}$ are the values of the local observable corresponding to measurement outcomes $a$ and $b$, respectively. As for the full measurement scenario, we may list the components of the joint observable to form a vector $\vec{c}$ in $\mathbb{R}^{D}$ with $D=M^{2}$ [compare $D=M^{2}\left(K^{2}-1\right)$ for the full probability distribution]. For deterministic protocols with at most $r$ bits of communication, the allowed functions $\alpha$ and $\beta$ are the same as in the previous section, but now correspond to vectors with components 
$c_{i, j}=A_{\alpha(i, j)} B_{\beta(i, j)}$. Since the map given by Eq. (5) is linear, the vectors corresponding to joint observables accessible using randomness remain convex combinations of the vectors accessible via deterministic protocols.

We now specialize to the scenario where each party has local \pm 1 -valued observables $(K=2)$ and they exchange $r=1$ bit of communication. The joint correlation observable then has components $c_{i, j}=p_{0,0 \mid i, j}+p_{1,1 \mid i, j}-p_{0,1 \mid i, j}-$ $p_{1,0 \mid i, j}$. If $M=2$, we obtain only trivial inequalities $-1 \leq c_{i, j} \leq 1$.

If $M=3$, the polytope has 320 extreme vectors. Using both the primal-dual algorithm and double description method for facet enumeration [15] we find that this polytope has 498 facets. 18 of these describe the trivial inequalities $-1 \leq c_{i, j} \leq 1$. The remaining 480 facets can be described by the inequalities

$$
\sum_{i, j=0}^{2} M_{i, j} c_{i, j} \leq 1
$$

where $M_{i, j}$ is either

$$
M_{1}=\frac{1}{6}\left(\begin{array}{ccc}
0 & -1 & 1 \\
-1 & 1 & 1 \\
1 & 1 & 1
\end{array}\right), \quad M_{2}=\frac{1}{11}\left(\begin{array}{ccc}
1 & 2 & -2 \\
2 & 1 & 2 \\
-2 & 2 & 1
\end{array}\right)
$$

or any matrix obtained from these two matrices by (i) permuting the rows and/or columns of the matrix and/or (ii) multiplying any subset of the rows and columns of the matrix by -1 . The full set of inequalities is a complete set of Bell joint observable inequalities for $M=3$.

Let us show that quantum theory satisfies all of the above Bell joint observable inequalities with auxiliary communication. We do this for a single one of the inequalities and the other inequalities all follow by a similar argument. For \pm 1 valued observables $\mathbf{A}_{i}$ and $\mathbf{B}_{j}$ and the joint quantum state $\rho$, a particular inequality looks like $\operatorname{Tr}\left[\rho \mathbf{T}_{i}\right] \leq 1$, where $\mathbf{T}_{i}$ is the operator corresponding to matrix $M_{i}$, e.g., $\mathbf{T}_{1}=\left[\mathbf{A}_{1}\left(-\mathbf{B}_{2}+\mathbf{B}_{3}\right)+\mathbf{A}_{2}\left(-\mathbf{B}_{1}+\right.\right.$ $\left.\left.\mathbf{B}_{2}+\mathbf{B}_{3}\right)+\mathbf{A}_{3}\left(\mathbf{B}_{1}+\mathbf{B}_{2}+\mathbf{B}_{3}\right)\right] / 6 . \operatorname{Tr}[\rho \mathbf{T}]$ is bounded by the sup norm of $\mathbf{T},|\mathbf{T}|=\sup _{|\psi\rangle}|| \mathbf{T}|\psi\rangle|| /|||\psi\rangle||$ and further $\operatorname{Tr}[\rho \mathbf{T}] \leq\left|\mathbf{T}^{k}\right|^{1 / k}$. Calculation of $\mathbf{T}^{k}$ yields a polynomial in $\mathbf{A}_{i}, \mathbf{B}_{i}$, and $\mathbf{I}$. Since $|\mathbf{X}+\mathbf{Y}| \leq|\mathbf{X}|+|\mathbf{Y}|$ and $|\mathbf{P}| \leq 1$ for any product $\mathbf{P}$ of $\mathbf{A}_{i}, \mathbf{B}_{j}$, and $\mathbf{I}$, it follows that $\left|\mathbf{T}^{k}\right|$ is less than or equal to the sum of the absolute value of the coefficients in the polynomial expansion of $\mathbf{T}^{k}$. By computer calculation we find that the sum of the absolute value of the coefficients of $\mathbf{T}_{1}^{4}$ is $\frac{155}{162}$ so that $\left|\mathbf{T}_{1}\right| \leq$ $\sqrt[4]{155 / 162}$. Thus this Bell inequality with auxiliary communication is satisfied. Similar arguments using $\mathbf{T}_{1}^{4}$ or $\mathbf{T}_{2}^{5}$ show that all of the inequalities Eq. (6) are satisfied. Therefore in the scenario where each party chooses one of three two-outcome measurements, a single bit of communication is sufficient to simulate the joint correlation observable in quantum theory for all quantum states and all quantum observables.
Conclusion. - Bell inequalities with auxiliary communication are a powerful new tool for understanding the cost of producing quantum correlations. Surprisingly, in all the cases we considered, it was sufficient to augment local realistic theories with a single bit of communication to simulate the quantum correlations. It remains a challenge to find a Bell inequality with auxiliary communication that is violated by a quantum state and set of quantum measurements [16].

We would like to acknowledge conversations with Allison Coates. This work was supported by the National Science Foundation under Grant No. EIA-0086038.

*Electronic address: dabacon@cs.caltech.edu

†Electronic address: toner@theory.caltech.edu

[1] P.W. Shor, in Proceedings of the 35th Annual Symposium on the Foundations of Computer Science, Santa Fe, NM, 1994 (IEEE Computer Society, Los Alamitos, CA, 1994), pp. 124-134; L. Grover, in Proceedings of the 28th Annual ACM Symposium on the Theory of Computing (ACM Press, New York, 1996), pp. 212-219.

[2] J. S. Bell, Physics (Long Island City, N.Y.) 1, 195 (1964).

[3] R. F. Werner and M. M. Wolf, Quantum Inf. Comput. 1, 1 (2001).

[4] G. Brassard, R. Cleve, and A. Tapp, Phys. Rev. Lett. 83, 1874 (1999); G. Brassard, quant-ph/0101005.

[5] T. Maudlin, in Proceedings of the 1992 Meeting of the Philosophy of Science Association, edited by D. Hull, M. Forbes, and K. Okruhlik (Philosophy of Science Association, East Lansing, MI, 1992), Vol. 1, pp. 404417; N. Cerf, N. Gisin, and S. Massar, Phys. Rev. Lett. 84, 2521 (2000); M. Steiner, Phys. Rev. A 270, 238 (2000); S. Massar, D. Bacon, N. Cerf, and R. Cleve, Phys. Rev. A 63, 052305 (2001); A. Coates, quant-ph/0203112.

[6] J.S. Bell, Speakable and Unspeakable in Quantum Mechanics (Cambridge Press, New York, 1993).

[7] B. F. Toner and D. Bacon, The Communication Cost of Simulating Bell Correlations, quant-ph/0304076 (to be published).

[8] M. Friossart, Nuovo Cimento Soc. Ital. Fis. 64B, 241 (1981); I. Pitowsky, Quantum Probability-Quantum Logic (Springer, Berlin, 1989);R. F. Werner and M. M. Wolf, Phys. Rev. A 64, 032112 (2001).

[9] E. Kushilevitz and N. Nisan, Communication Complexity (Cambridge University Press, New York, 1997).

[10] R. T. Rockafellar, Convex Analysis (Princeton University Press, Princeton, New Jersey, 1970).

[11] A. Fine, Phys. Rev. Lett. 48, 291 (1982).

[12] I. Pitowsky and K. Svozil, Phys. Rev. A 64, 014102 (2001).

[13] A. Garg and N. D. Mermin, Found. Phys. 14, 1 (1984).

[14] A. C. Yao, in Proceedings of the 11th ACM Symposium on Theory of Computing, Atlanta, GA, 1979 (ACM Press, New York, 1979), pp. 209-213.

[15] D. Bremner, K. Fukuda, and A. Marzetta, Disc. Comput. Geom. 20, 333 (1998); K. Fukuda and A. Prodon, Lect. Notes Comput. Sci. 1120, 91 (1996).

[16] That such a violation must exist is implied by [4]. 